Article

\title{
Minimum-Variance Control System with Variable Control Penalty Factor
}

\author{
Ioan Filip, Florin Dragan, Iosif Szeidert * $*$ and Adriana Albu(D) \\ Department of Automation and Applied Informatics, Politehnica University of Timisoara, Bvd. V. Parvan, No. 2, \\ 300223 Timisoara, Romania; ioan.filip@aut.upt.ro (I.F.); florin.dragan@aut.upt.ro (F.D.); \\ adriana.albu@aut.upt.ro (A.A.) \\ * Correspondence: iosif.szeidert@aut.upt.ro; Tel.: +40-0256-403237
}

Received: 18 February 2020; Accepted: 25 March 2020; Published: 27 March 2020

check for updates

\begin{abstract}
The present paper proposes (as the main contribution) an additional self-tuning mechanism for an adaptive minimum-variance control system, whose main goal is to extend its functionality for a large value range of unmeasurable perturbations which disturb the controlled process. Through the standard design procedure, a minimum variance controller uses by default an internal self-tuning mechanism based on the process parameter estimates. However, the main parameter which overwhelmingly influences the control performance is the control penalty factor $(\rho)$. This parameter weights the term that describes the control variance in a criterion function whose minimization is the starting point of the control law design. The classical minimum-variance control involves an off-line tuning of this parameter, its value being set as constant throughout the entire operating regime. Based on the measurement of the process output error, the contribution of the proposed strategy consists in a real-time tuning of the control penalty factor, to ensure the stability of the control system, even under conditions of high disturbances. The proposed tuning mechanism adjusts this parameter by implementing a bipositional switching strategy based on a sharp hysteresis loop. Therefore, instead of the standard solution that involves a constant value of the control penalty factor $\rho$ (a priori computed and set), this paper proposes a dual value for this controller parameter. The main objective is to allow the controlled process to operate in a stable fashion even in more strongly disturbed regimes (regimes where the control system becomes unstable and is usually switched off for safety reasons). To validate the proposed strategy, an induction generator integrated into a wind energy conversion system was considered as controlled plant. Operating under the action of strong disturbances (wind gusts, electrical load variations), the extension of safe operating range (thus avoiding the system disengagement) is an important goal of such a control system.
\end{abstract}

Keywords: adaptive control; minimum-variance controller; control penalty factor; self-tuning strategy; induction generator

\section{Introduction}

The usage of adaptive control systems is suitable for the control of complex systems for which an accurate mathematical model is not available, with the system being subject to unknown parameter variations and large disturbances over time [1-6]. Furthermore, if the operating point changes under the action of external disturbances (taking into account a wide variation range of disturbances) or due to the variation of internal parameters, the usage of classical control solutions (PI, PID controllers) becomes infeasible [5,7]. In these cases, a minimum variance control system is a viable solution due to its adaptive nature, ensured by the self-tuning characteristic of the controller parameters [8-10]. Obviously, with these parameter estimates computed in real-time, a parameter estimator is required (as a necessary component integrated into the control system) [11-14]. 
Based on the minimization of a cost function described as a sum of two quadratic terms, the goals of the designed control law are the minimization of the controlled output variance, simultaneously with the minimization of the controller output variance (see relation 1, where $E$ is an expectation operator) [15-18]:

$$
J=E\left\{[\text { process output variance }]^{2}+\rho[\text { controller output variance }]^{2}\right\}
$$

The second mentioned goal (leading to a suboptimal control) is a mandatory condition to ensure the possibility of practical implementation, imposing a penalty on the control variance, so that the computed control values to be physically achievable [15-19]. The balance between these two objectives of the cost function is achieved through a control penalty factor $(\rho)$, which weights the significance of the second quadratic term in the criterion function. The technical literature states that, theoretically, the control penalty factor $\rho \in[0,1]$, but in practice it is usually set in the range $\rho \in[0.0001,0.1]$, ensuring a proper weighting between the minimization of the system output variance and the control variance [1-3]. A null value of the control penalty factor $(\rho=0)$ would hypothetically lead to an optimal control law, only theoretically feasible, its implementation in practice being impossible. A simple analysis of such an optimal control system shows that huge control values are generated, but these values are not achievable in practice. Therefore, a non-zero value for the $\rho$ factor is a mandatory condition to ensure a practical implementation of a minimum variance control system. A smaller control penalty factor leads to a higher control variance (which can sometime destabilize the system) and, as a consequence, to more efficient minimization of the controlled output variance (ensuring good control performance). A higher $\rho$ parameter leads to a smaller control variance and to a weaker penalization of the system output variance (which can negatively affect the control performance). However, a too low penalization of the control is the main cause that can destabilize the control system. A proper value of $\rho$, neither too small nor too big, could be a compromise solution [12,16,17].

The basic idea of the proposed control strategy is that, instead of a constant value of the control penalty factor $\rho$ (a priori set), an algorithm can be designed to on-line tune its value in accordance with the operating conditions of the control system. Even though the self-tuning feature of the minimum variance controller is implicitly ensured by using real-time estimates as parameters of the control law, the control penalty factor is the main parameter that decisively influences the performance of the control system (including the system stability). The main goal of the present paper is to design an algorithm to real-time tune the control penalty factor, while simultaneously ensuring the system stability and good control performance (for a large variation range of the unmeasurable perturbations which can disturb the controlled process).

For a control system having as its main objective the rejection of the disturbance effect, two situations can be considered, depending on the possibility of measuring the disturbance. For the case when the disturbance is directly measurable, a strategy for on-line tuning of the control penalty factor was described by the authors in [17]. For the case when the disturbance is not directly measurable, a tuning strategy can be designed by considering an indirect measurement of disturbance, more precisely, by measuring the disturbing effect produced by it (this being the subject of this paper). In order to validate the proposed control strategy, an induction generator connected to a power system was considered as a controlled plant. An induction generator is a highly nonlinear plant (described by a mathematical model of the seventh order) and its operating point is frequently changing under the action of internal or external disturbances [20-24]. In this case, an electrical load/unload is considered one of the main disturbances whose effect must be rejected by the system. Such a disturbance cannot be directly measured. However, its effect on the process output can be measured. For any type of disturbance, this effect translates into the error of the controlled output. Due to this reason, the proposed strategy must be tested for any disturbance (measurable or unmeasurable) that produce an output error. For the case of an induction generator chosen as a controlled process, the mechanical torque variation and the load/unload regimes are the considered disturbances. 
An extension of the value range of disturbances, whose effect can be rejected, is the major objective of the new proposed control algorithm, leading to an increase of the control system stability and robustness. Although the validation of this improved control strategy will be performed on this particular case (induction generator), its logic is applicable for any minimum variance control of other complex plant.

\section{Design of the Minimum Variance Control Law}

It is common that the design of the minimum variance control law starts with the assumption of a linearized model of the controlled plant, which describes its functionality around an operating point accurately enough $[1,3]$. The behavior of a nonlinear plant can be described in the vicinity of an operating point by such a linearized model of a certain order, with parameters computed by a parameter estimator. A change of the operating point has as consequence a change of the linear model's parameters and the real-time tracking of these can be done by such a parameter estimation algorithm (in this case, the recursive least square estimator-RLS) [12-14]. For the case of an induction generator connected to a power system, its complete functionality is described by a nonlinear mathematical model of the seventh order (the $d-q$ model represented by the Park equations) $[10,22,23]$. The complete model (and the parameter values) of the considered process has already been presented in extenso in one of the author's recent paper [10] and therefore it is not detailed in the present paper (being mentioned only in an Appendix A at the end of the paper).

The goal of the proposed control system is to maintain constant the voltage at generator terminals through the excitation voltage control, considering the plant perturbed by various external disturbances (electrical consumer load/unload, mechanical torque variations).

The technical literature shows that this type of relation between the excitation voltage (considered as the plant input variable) and the terminal voltage (as plant output variable) can be enough accurately described by a 4 th order linear mathematical with time-varying parameters $[12,22,24,25]$. This reduced order (4th) is based on several simplifying assumptions regarding the electrical phenomenology, which does not affect the accuracy of the model near an operating point $[12,15,26]$. For maximum accuracy of the simulation results, the complete 7th order nonlinear model of the controlled process was used to test the proposed control strategy. It should be noted that this simplified linear model was used only for the design stage of the control law. Therefore, the starting point of the control law design is a linear model described by a 4th order discrete difference equation (see Equation (2)):

$$
A\left(q^{-1}\right) y_{t}=q^{-1} B\left(q^{-1}\right) u_{t}
$$

where

$$
\begin{gathered}
A\left(q^{-1}\right)=a_{4} q^{-4}+a_{3} q^{-3}+a_{2} q^{-2}+a_{1} q^{-1}+1 \\
B\left(q^{-1}\right)=b_{3} q^{-3}+b_{2} q^{-2}+b_{1} q^{-1}+b_{0}
\end{gathered}
$$

and $y_{t}$ is the terminal voltage (controlled output at discrete time $t$ ), $u_{t}$ is the excitation voltage (controller output), $q^{-1}$ is the shift operator (with one sampling time, therefore $y_{t-1}=q^{-1} y_{t}$, and so on), $a_{1, \ldots, 4}$ $b_{0, \ldots, 3}$ are parameters of polynomials $A\left(q^{-1}\right)$ and $B\left(q^{-1}\right)$.

This equation is valid around an operating point for a set of parameter values and around another operating point for other set of parameter values (the tracking of these parameters being performed by the parameter estimator).

The control law design involves minimizing a cost function described by relation (4) [15-18]:

$$
J=E\left\{\left[y_{t+1}-w_{t}\right]^{2}+\rho\left[u_{t}-u_{t}^{*}\right]^{2}\right\}
$$

where $y_{t+1}$ is the process output at discrete time $t+1$ (next sampling time); $u_{t}$ is the controller output, $u_{t}^{*}$ is the steady state controller output; $w_{t}$ is the set point, $\rho$ is the control penalty factor; and $E\{$.$\} is the$ expectation (mean operator). 
Minimizing the criterion function (4) (by solving the equation: $\frac{d J}{d u_{t}}=0$ ) and taking into account that, in steady state regime: $q^{-1}=1, u_{t}^{*}=\frac{\hat{A}(1)}{\hat{B}(1)} w_{t}$, respectively $w_{t}=y_{t}$, the following control law results [17]:

$$
u_{t}=\frac{-q\left[1-\hat{A}\left(q^{-1}\right)\right]}{\hat{B}\left(q^{-1}\right)+\rho} y_{t}+\frac{1+\rho \frac{\hat{A}(1)}{\hat{B}(1)}}{\hat{B}\left(q^{-1}\right)+\rho} w_{t}
$$

where $\hat{A}$ and $\hat{B}$ are the estimates of $A$ and $B$ polynomials (calculated by the RLS estimator).

The control law described by relation (5) will be used for all performed tests to validate the proposed control strategy. The adaptive feature of such a controller is highlighted by the presence of parameter estimates (time-varying) in the control law (5). In case of RLS estimator, these estimates depend on the forgetting factor $\lambda$. However, the influence of this factor over the control system perturbed by external disturbances is quite low $[18,25,27,28]$. The influence of this tuning parameter $(\lambda)$ is much more significant in the case of process with time-varying internal parameters $[10,12,18,29]$. Therefore, a constant value (off-line properly set) is used during the entire operating regime of the process. The plant is considered disturbed by a stochastic noise (with zero mean and variance $\sigma^{2}=0.01$ ), as a mandatory condition for a proper functioning of the RLS estimator (ensuring the numerical stability) $[16,27,30-32]$. Also, a constant value $\lambda=0.995$ is identified and set for the forgetting factor, providing the best results.

Instead, the control penalty factor $(\rho)$ is the tuning parameter that has a much greater influence over the control system performance and stability [12,16,32]. Therefore, the adaptive feature of the minimum variance controller could be enhanced by implementation of an additional self-tuning mechanism, which allows a real-time adjustment of this control penalty factor $(\rho)$. In other words, instead of a constant $\rho$, the system will operate with a variable $\rho$. The proposed control strategy is based on the observations that these extreme values of $\rho$ (very small or very big) each have their own advantages:

- A high value of $\rho$ ensures a better penalty of the excessive control (and a better system stability), to the detriment of qualitative output performance;

- A small value of $\rho$ can ensure better qualitative performance of the controlled output, but with the risk of destabilizing the control system (since the control is much less penalized).

By considering both situations, the main goal of this paper is to find a compromise solution that can solve the problem, requiring the design of an adequate controller tuning algorithm, which will be presented in the next paper section.

Regarding the stability of the minimum-variance control system, a complete analysis was carried out by authors in other papers $[10,11,16,17]$.

\section{The Self-Tuning Algorithm of Control Penalty Factor}

Based on the above presented considerations, the self-tuning algorithm of the control penalty factor $(\rho)$ considers two possible switching values $\rho_{0}$ and $\rho_{1}$. The question that needs to be answered is: what are the conditions to switch $\rho$ from one value to another? Thus, two switching conditions are required to be set:

- $\quad$ The condition for switching from the low value $\rho_{0}$ to the high value $\rho_{1}$;

- The return condition, for switching from the high value $\rho_{1}$ to the low value $\rho_{0}$.

Also, related to these two possible values of $\rho$, another question that requires an answer is: which of the two values of $\rho$ is chosen as a fixed value for the steady-state regime (undisturbed regime)? The answer is the lower value $\rho_{0}$, which provides the best performance for small disturbances, while stabilizing the control system. Thus, the switch to the high value $\rho_{1}$ triggers only when a major disturbance occurs. 
Starting with the assumption that the disturbance is not measurable, the only variable indicating their random action is the controlled output error. In other words, the activation of this switching mechanism is triggered based on the output error value. Obviously, a high error indicates a stronger disturbance and a lower error indicates a weaker disturbance. In this last case, a low value of $\rho\left(\rho_{0}\right)$ can provide both stabilization and good performance of the control system. If the output error exceeds a certain set threshold, the system stabilization requires a higher value of $\rho\left(\rho_{1}\right)$, in order to strongly penalize the excessive control. However, by keeping this high value $\left(\rho_{1}\right)$ set a too long time period can excessively penalize the control, which becomes too weak to ensure the control system stability $[16,17]$. Therefore, both a too long-time strong control and a too weak control can destabilize the control system if it is severely disturbed. The compromise solution is to set a small constant value for $\rho\left(\rho_{0}\right)$ in steady-state regime, respectively, when the error exceeds a certain threshold, to switch to a high value $\left(\rho_{1}\right)$ for a limited time period. This limited time period should last until the output error drops below another certain set threshold, thus avoiding the destabilization of the control system. Obviously, it becomes imperative to find and set these switching thresholds of the control penalty factor between these two imposed values $\left(\rho_{0}, \rho_{1}\right)$.

A viable solution requires a sudden (sharp) change of $\rho$ to the maximum level $\rho=0.1$ (at the critical time moment when a high disturbance occurs) for an efficient control penalty and, implicitly, for a control system stabilization. A logic algorithm based on a sharp hysteresis loop (with two switching levels) can implement such a tuning strategy (see Figure 1). Such a simple and efficient algorithm requires finding the appropriate level values $\rho_{0}$ and $\rho_{1}$ and, obviously, the error thresholds $e 1$ and $e 2$ where the control penalty factor $(\rho)$ switches between these two levels $\left(\rho_{0}, \rho_{1}\right)$. One of the goals of the case studies presented below is to find these proper parameters values (levels $\rho_{0} / \rho_{1}$; thresholds e1/e2 - see Figure 1) in order to implement, test and validate the proposed control strategy, integrating a tuning algorithm based on a sharp hysteresis loop.

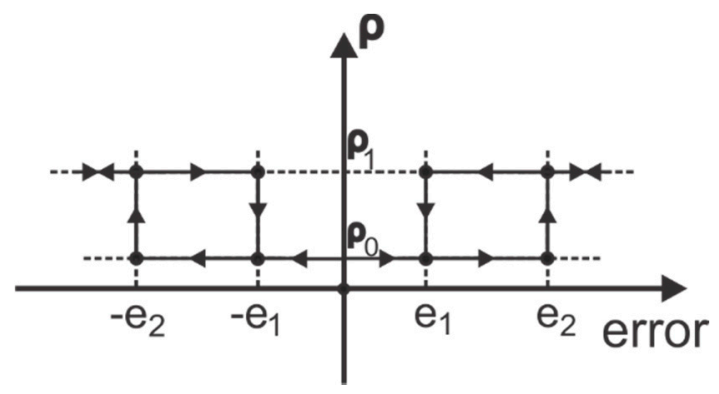

Figure 1. The self-tuning mechanism of the control penalty based on a sharp hysteresis loop.

Taking into consideration such proposed real-time self-tuning mechanism of the control penalty factor, the general structure of the minimum variance control system is presented in Figure 2. It can be seen in this figure that the controlled output error (terminal voltage error) is the input of the hysteresis block, which is the core of the proposed self-tuning algorithm. In this context, the "normal" state of the system is the stationary regime (when the process is not disturbed or is disturbed only by a low stochastic noise) or a regime in which the system is weakly disturbed by small external disturbances. In both cases, the proposed adjustment mechanism for tuning the control penalty factor is not active, the control penalization being set to lowest value of $\left(\rho=\rho_{0}=0.0001\right)$. 


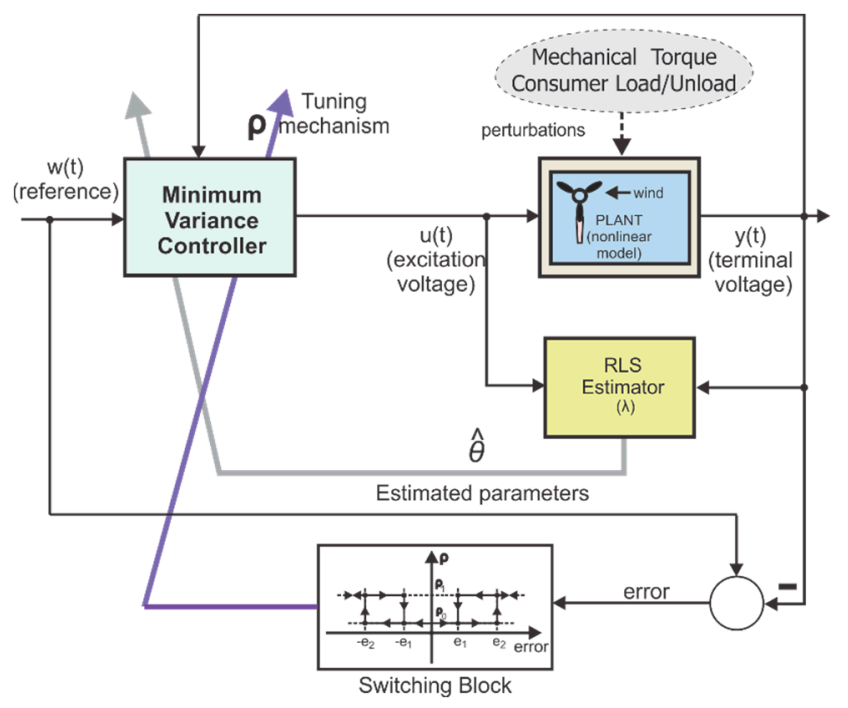

Figure 2. General structure of the proposed self-tuning minimum variance control system.

Based on simulations results analysis, the calibration procedure that was followed to determine the two thresholds of the error when the control penalty factor $(\rho)$ switches between the two levels $\left(\rho_{0}\right.$, $\rho_{1}$ ), involves the following steps:

1. The minimum variance control system is started (without enabling the proposed self-tuning switching mechanism), setting a constant maximum penalty of control $(\rho=0.1)$ and considering the maximum allowed value of the disturbance for which it remains stable and the control performance is good;

2. The maximum value of the system output error (the overshoot) is determined to be used as an initial benchmark for setting the high switching threshold $e 2$;

3. The value of the high switching threshold $e 2$ is set about $10 \%$ lower than the maximum value of the output error previously determined.

4. Also, as an initial benchmark, the value for the low threshold $e 1$ is set with an order of magnitude larger than the error produced in stationary regime by the stochastic noise that affects the process (needed to ensure the numerical stability of the parameters estimator).

5. Keeping the same disturbance, the control system is restarted, enabling the proposed self-tuning switching mechanism, initially calibrated with the values $e 1$ and $e 2$ previously established (analyzing the control system stability and its performances: the settling time, the transient regime duration).

6. Small adjustments around these values $e 1$ and $e 2$ can be performed to obtain better results based on the observations that fine changes of the high switching threshold $e 2$ can affect the control stability and fine changes in the low switching threshold e1 can affect the control performance (the settling time or the duration of the transient oscillating regime).

7. Finally, larger perturbations are applied and step 6 is resumed, fine adjustments of these two thresholds $(e 1, e 2)$ being carried out successively until the result is considered appropriate (stable system and good control performance).

8. The procedure stops when, for a very high disturbance, an adequate set $(e 1, e 2)$ can no longer be determined to ensure the stability of the system (this disturbance being considered the maximum limit for the control system operating range).

Comparing the solution described by the authors in [17] with the one presented in this paper, there are some major differences. Thus, when a high measurable disturbance occurs, the algorithm presented in [17] involves one single rectangular pulse variation of the control penalty factor, with a fixed duration (previously well-established) and the method can be applied only for the case of 
measurable disturbances. The presented strategy can involve multiple rectangular pulse changes of the control penalty factor (with a variable duration based on a logic algorithm using a sharp hysteresis loop) and (additionally) can be applied also for unmeasurable disturbances (for example, the unpredictable load/unload regimes), requiring only a measurement of the controlled output. Also, in [10], the authors describe some classical minimum variance control systems (with a constant penalty control factor), analyzing the control performance for different orders of the induction generator linearized model (used only as a starting point to design the control law). A starting procedure of a minimum variance control system, involving successively different values of the control penalty factor, is presented in [16]. A relatively similar solution, proposing a self-tuning algorithm for controller output singletons of an adaptive fuzzy PI control system, has been implemented and the results were published by the authors in [7], noting comparably good performances. The theoretical aspects and the full calculus regarding the analytical determination of the minimum-variance control law were described in extenso in [11,12]. The performances of these referred control structures are comparable, each of them having its own functional and applicability particularities.

\section{Case Studies to Validate the Self-Tuning Algorithm}

For the considered process, an induction generator integrated into a wind energy conversion system, high variations of the wind speed (produced by wind gusts), over a certain limit, may require the disengagement of the energy conversion system to protect it (for example, by furling the wind turbine). Also, a strong disturbance produced by a high electrical load/unload can activate the protections that disengage the system. Any solution that can increase the allowed value range of disturbances extends the functionality domain of the controlled process. Therefore, maintaining the functional capabilities of the control system (energy production, energy supply to consumers), even under harsher conditions caused by highly disturbances, is the main goal of the proposed strategy.

Any disturbance that acts on the system causes a process output error (this being the main indicator that signals a disturbance). For this reason, the next tests will target both the load/unload regime (when the disturbance is not directly measurable) and the case of a mechanical torque variation (although this could be also directly measured, but an output error occurs anyway). The mechanical torque variation is an effect of wind speed variation, considering the generator integrated into a wind energy conversion system. The load/unload regime means a variation of the electrical admittance at generator terminal, as effect of consumers connecting/disconnecting.

The mechanical torque $T_{m}$ (its variation being seen as a disturbance) is a parameter referred in the equation describing the mechanical motion within the induction generator mathematical model. Also, the connection between the induction generator and the power system includes a local electrical consumer connected at generator terminals, so the equations describing the terminal voltage projections of the stator load winding $d-q$ axis integrates the consumer electrical admittance, also considered as an internal parameter whose variation disturbs the system. The complete model of the dual fed induction generator (DFIG) connected to a power system (PS) through a long transmission line is presented in the Appendix A [10]. For different values of the control penalty factor $(\rho)$, the control system performance and the stability will be analyzed in order to find the maximum allowed perturbation for which the process can still operate.

The first study case takes into consideration a mechanical torque variation as an external perturbation produced by wind gusts.

(a) Mechanical torque variation $\left(\Delta T_{m}\right)$

For several tuning values of $\rho$, Table 1 concisely shows the results of performed tests, highlighting the control performance. 
Table 1. The case studies—results for maximum allowed values of mechanical torque variation $\left(\Delta T_{m}\right)$.

\begin{tabular}{|c|c|c|}
\hline$\rho$ & $\Delta T_{m}$ & Control Performance \\
\hline$\rho=0.0001$ (constant) & $\Delta T_{m} \leq 16 \%$ & $\begin{array}{l}\text { Stable system and very good control performances } \\
\text { (settling time: } 0.2 \mathrm{~s} \text { ) }\end{array}$ \\
\hline$\rho=0.001$ (constant) & $\Delta T_{m} \leq 16 \%$ & $\begin{array}{l}\text { Stable system and good control performances } \\
\text { (settling time: } 0.5 \mathrm{~s} \text { ) }\end{array}$ \\
\hline$\rho=0.01$ (constant) & $\Delta T_{m} \leq 16 \%$ & $\begin{array}{l}\text { Stable system and satisfactory control performances } \\
\text { (settling time: over } 1 \mathrm{~s} \text { ) }\end{array}$ \\
\hline$\rho=0.1$ (constant) & $\Delta T_{m} \leq 18 \%$ & $\begin{array}{l}\text { Stable system but poor control performances } \\
\text { (settling time: over } 5 \mathrm{~s} \text { ) }\end{array}$ \\
\hline$\rho \in\{0.0001,0.1\}$ (variable) & $\Delta T_{m} \leq 24 \%$ & $\begin{array}{l}\text { Stable system and very good control performances } \\
\text { (settling time: under } 0.5 \mathrm{~s} \text { ) }\end{array}$ \\
\hline
\end{tabular}

For a set of smaller values of the control penalty factor $(\rho=0.0001, \rho=0.001, \rho=0.01)$, the controlled output is depicted in Figure 3a-c. For all these three values of the control penalty factor, the maximum disturbance for which the control system remains stable is $\Delta T_{m}=16 \%$. A higher variation of the mechanical torque, over this threshold $\left(\Delta T_{m}>16 \%\right)$, leads to an unstable system due to the huge values of a weakly penalized control. Also, a degradation of the control performance can be noticed with the increase of the control penalty. Thus, the settling time successively increases and the transient regime is longer (see Table 1). The best performance is obtained for $\rho=0.0001$ (settling time $=0.2 \mathrm{~s}$, overshoot approx. $4 \mathrm{~V}$ ), while for $\rho=0.001$, the settling time is over $0.5 \mathrm{~s}$, respectively, and for $\rho=0.01$, it is over $1 \mathrm{sec}$.

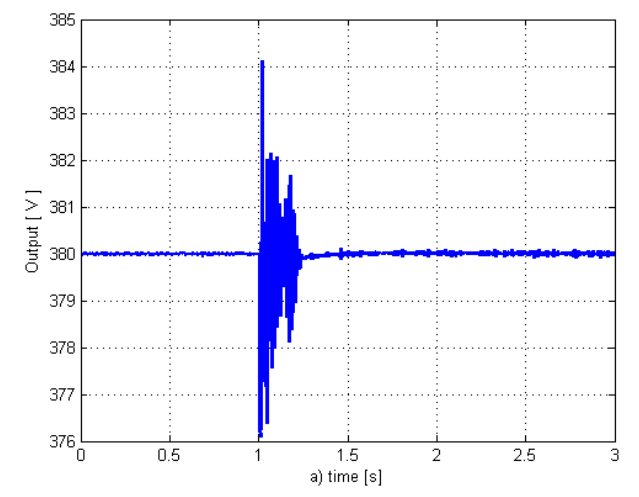

(a)

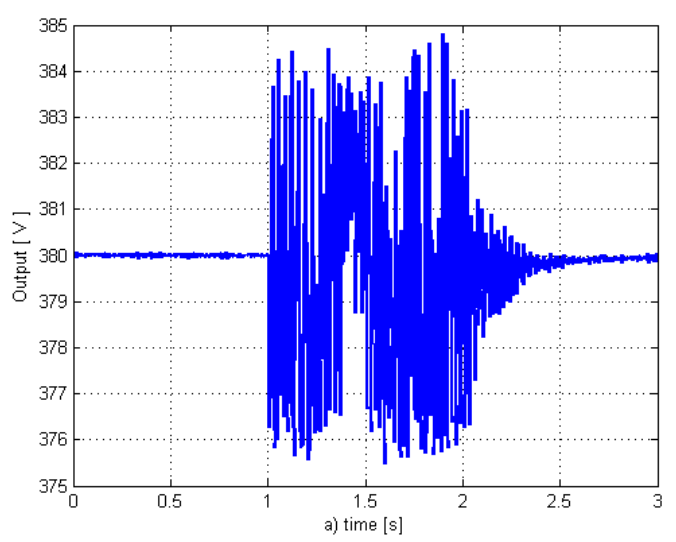

(c)

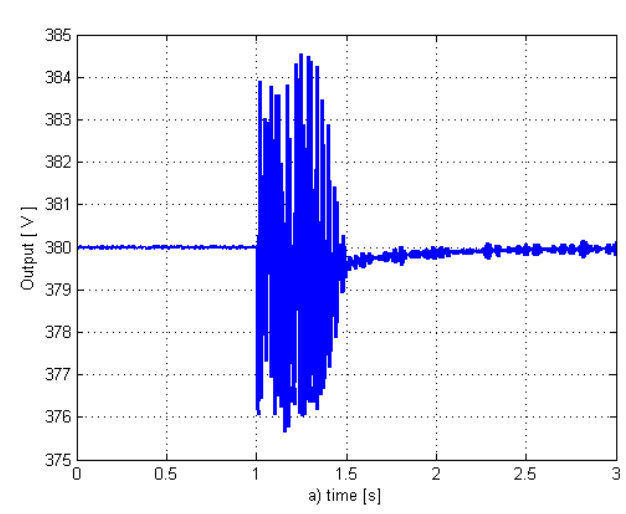

(b)

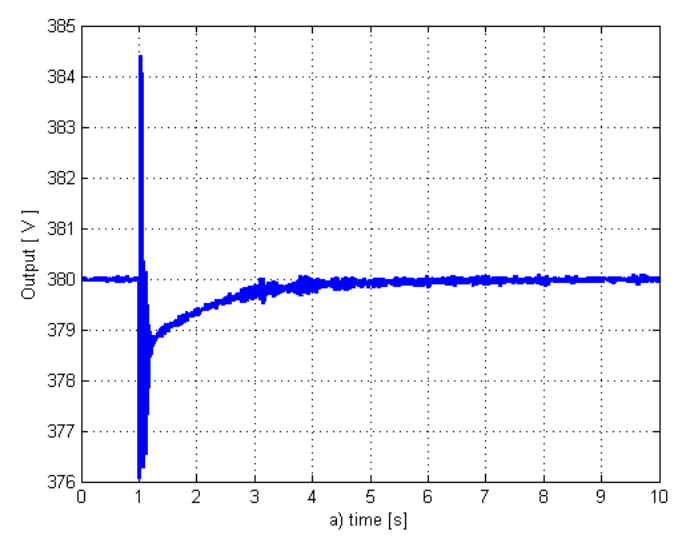

(d)

Figure 3. Cont. 


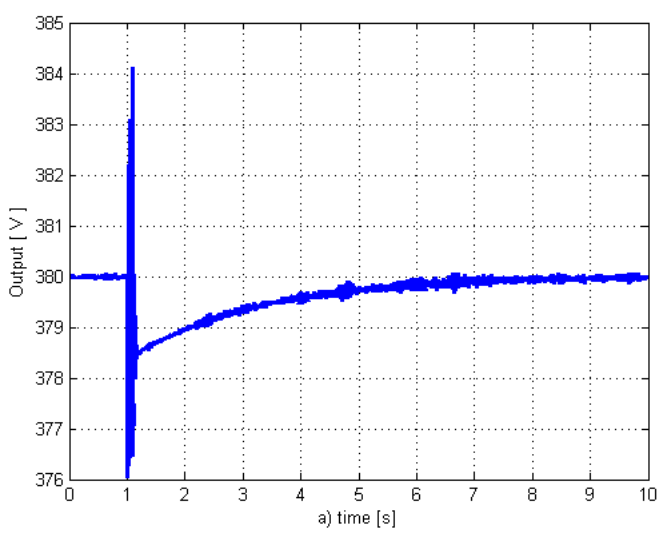

(e)

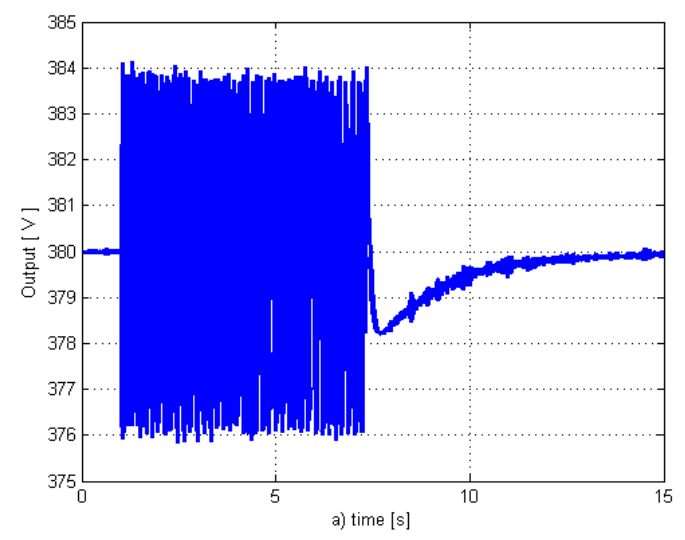

(f)

Figure 3. (a) Controlled output (terminal voltage)—constant $\rho=0.0001\left(\Delta T_{m}=16 \%\right)$; (b) Controlled output (terminal voltage)—constant $\rho=0.001\left(\Delta T_{m}=16 \%\right)$; (c) Controlled output (terminal voltage)—constant $\rho=0.01\left(\Delta T_{m}=16 \%\right)$; (d) Controlled output (terminal voltage)—constant $\rho=0.1$ $\left(\Delta T_{m}=16 \%\right) ;(\mathbf{e})$ Controlled output (terminal voltage)—constant $\rho=0.1\left(\Delta T_{m}=18 \%\right) ;(\mathbf{f})$ Controlled output (terminal voltage)—constant $\rho=0.1\left(\Delta T_{m}=19 \%\right)$.

The results are changed by considering an increase by an order of the control penalty factor ( $\rho=0.1$, see Figure $3 \mathrm{~d}-\mathrm{f}$ ). For the same maximum allowed disturbance as in the previous cases $\left(\Delta T_{m}=16 \%\right)$, the control performance is weaker, but the system remains stable (see Figure $3 \mathrm{~d}$ ). Although a significant attenuation of the oscillations can be noticed, the settling time exceeds $2 \mathrm{~s}$, and therefore the control system responds slower. However, in this case, for higher disturbances $\left(\Delta T_{m}=18 \%, \Delta T_{m}=19 \%\right)$, the major change consists in the fact that the system remains stable (see Figure 3e,f). This stabilization was not possible for the previous lower values of the control penalty. For $\Delta T_{m}=18 \%$, the settling time increases over 5 seconds and the control system response could still be considered acceptable (Figure 3e). For $\Delta T_{m}=19 \%$ the control system remains stable, but an unacceptably long transient regime (over $6 \mathrm{~s}$ ) and a too long settling time (over $10 \mathrm{~s}$ ) can be noticed (Figure 3f). Similar results are obtained for a disturbance over this value. The conclusion is that a high value of $\rho$ can stabilize the control system, even when it is highly disturbed, but the control performance is far too weak to be considered acceptable (too long settling time and oscillating regime). It should be noted that in all performed tests, the overshoot is relatively constant (slightly over 4 volts) due to the constraint imposed by the connection to a power system (which tries to maintain the generator terminal voltage at the constant value of power system).

Therefore, a comparative analysis of the extreme cases of control penalty setting $(\rho=0.0001$ and $\rho=0.1$ ) leads to the following conclusions (which are the starting point for the design of the proposed self-tuning algorithm):

- $\quad$ A small value of $\rho$ ensures a very good performance, but only for small disturbances;

- $\quad$ A high value of $\rho$ ensures a system stabilization even for higher disturbances, but with the cost of an unacceptable control performance degradation.

Taking into consideration the previous results, the proposed tuning strategy uses the following rules by implementing an algorithm based on a sharp hysteresis curve (Figure 1):

- In the stationary regime, when the output error is under a certain threshold $\mathrm{e}_{2}$, the control penalty factor $\rho$ is set to a low value $\left(\rho_{0}=0.0001\right)$ that ensures the best control performance.

- When a high disturbance occurs and, as a consequence, the output error passes over the threshold $\mathrm{e}_{2}$ (see Figure 1), the control penalty factor is set to a high value $\rho_{1}=0.1$, in order to stabilize the system through a strong penalty of control. Such a high value of $\rho$ can ensure the stability of the control system, even when it is highly disturbed. 
- When the output error falls below another fixed threshold $\mathrm{e}_{1}$ (so, after a limited time period), the control penalty factor returns to the low value $\rho_{0}=0.0001$, decreasing the control penalty and allowing good control performances, especially a small settling time. Therefore, this constant low value of $\rho$ is considered as a stationary regime value, being increased only temporarily when a high disturbance acts on the system and, as a consequence, whenever the controlled output error exceeds a certain threshold.

Based on the previous consideration, the next case study will test the proposed tuning strategy implementing the previous rules by using a bipositional switch with hysteresis (see Figures 1 and 2). The following parameters must be set: the error values for the switching thresholds $\left(e_{1}\right.$ and $\left.e_{2}\right)$ and the switching levels of the control penalty factor $\left(\rho_{0}\right.$ and $\left.\rho_{1}\right)$. In this case, the values of these tuning parameters (determined by multiple tests and ensuring the best results) are: $e_{1}=0.5, e_{2}=3.5$, $\rho_{0}=0.0001$ and $\rho_{1}=0.1$.

The first test considers a higher variation of the mechanical torque $\Delta T_{m}=17 \%$, for which none of the constant values of $\rho$ (see previous cases) can ensure the stability of the control system. The control system response (the terminal voltage) is shown in Figure 4a. Very good performance can be noticed (comparable to those obtained for $\rho_{0}=0.0001$-see Figure 3a): small settling time (under $0.5 \mathrm{~s}$ ), short oscillating transient regime ( $0.2 \mathrm{~s}$.). Also, the control variance is sufficiently penalized (the control values being feasible- see Figure $4 \mathrm{~b}$ ) and the estimates are numerically stable (Figure $4 \mathrm{~d}$ ). For such a higher disturbance $\left(\Delta T_{m}=17 \%\right)$, the proposed tuning mechanism is automatically triggered (the output error exceeding the threshold $e_{2}$ - see Figure 1). As a consequence, an adequate adjustment of the control penalty factor $(\rho)$ is performed (Figure 4c). At first (for a high output error), a high value $\rho_{2}=0.1$ is set, with the goal to strongly penalize the control (Figure $4 \mathrm{~b}$ ), thus avoiding the system destabilization. When the output error falls below the certain threshold $\left(e_{1}\right.$-see Figure 1$)$, the control penalty factor returns to the small stationary value $\rho_{1}$, allowing a reasonable increase of the control to ensure a reduced settling time, a short oscillating transient regime and, thus, the control system performances are very good (Figure $4 a$ ).

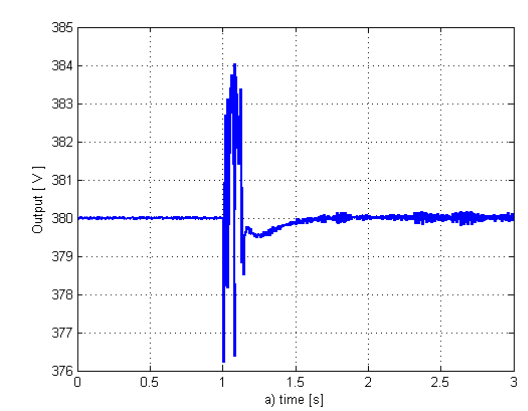

(a)

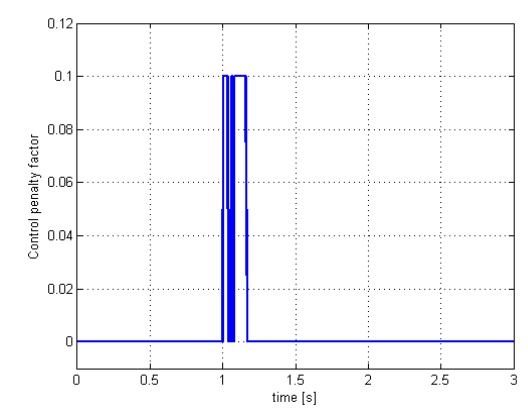

(c)

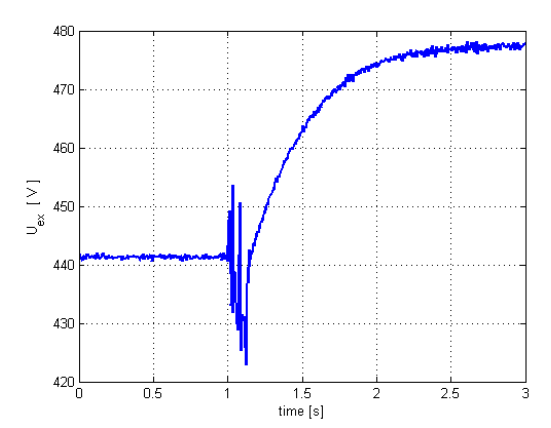

(b)

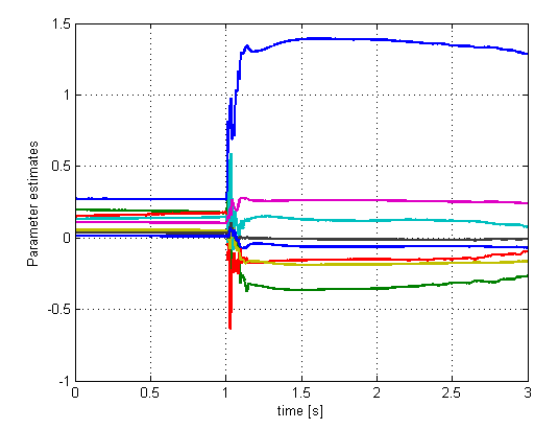

(d)

Figure 4. (a) Controlled output (terminal voltage) $-\rho \in\{0.0001,0.1\}\left(\Delta T_{m}=17 \%\right)$; (b) Controller output (excitation voltage) $-\rho \in\{0.0001,0.1\}\left(\Delta T_{m}=17 \%\right)$; (c) Variable setting of control penalty factor $-\rho \in\{0.0001,0.1\}\left(\Delta T_{m}=17 \%\right)$; (d) Parameters estimates. 
The maximum value of the disturbance for which the enhanced control system operates stable is $\Delta T_{m}=24 \%$ (see Figure 5a). The controlled output has a very good settling time and a short transient regime (under $0.5 \mathrm{~s}$ ). Also, the tuning mechanism of the control penalty factor is activated for a longer period of time, as in the previous case (Figure $5 b)$. Over this disturbance value $\left(\Delta T_{m} \geq 25 \%\right)$, the control system becomes unstable.

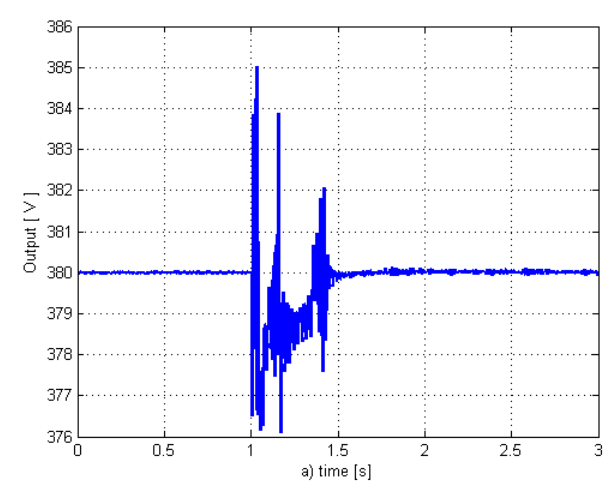

(a)

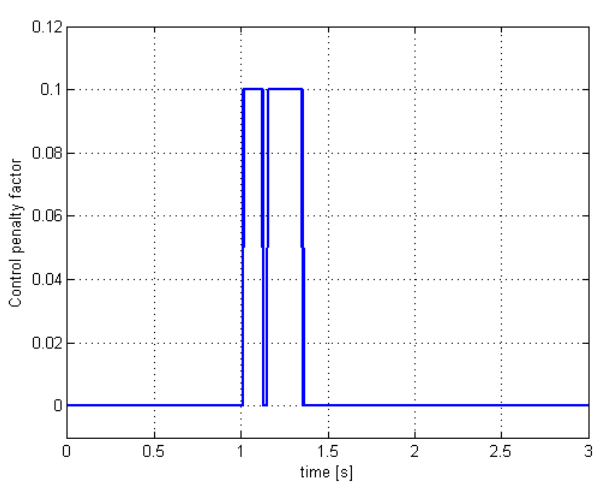

(b)

Figure 5. (a) Controlled output (terminal voltage)—constant $\rho \in\{0.0001,0.1\}\left(\Delta T_{m}=24 \%\right.$ ); (b) Variable setting of control penalty factor.

In conclusion, the proposed control strategy allows an extension of the values' range of disturbance (mechanical torque) up to 50\%, from $\Delta T_{m}=16 \%$ (the maximum allowable disturbance for which a small constant $\rho=0.0001$ still provides good control performance and system stability-see Figure 3a) up to $\Delta T_{m}=24 \%$ (the maximum allowable disturbance for which, only by using the proposed tuning mechanism with a variable control penalty factor $\rho \in\{0.0001,0.1\}$, the control system remains stable and has good control performances- see Figure 5a).

Following this, a similar suite of tests is carried out for the second type of disturbance (the variation of the electrical resistance at the generator terminals, caused by an electrical load/unload).

(b) Electrical load (electrical resistance variation $\Delta T_{m}$ )

As in the previous case (mechanical torque variation), the next performed tests consider the same four values of the control penalty factor. Figure $6 a-d$ present the obtained results for each test and the control performances are concisely shown in Table 2.

Table 2. The case studies—results for maximum allowed values of electrical load variation $(\Delta R)$.

\begin{tabular}{|c|c|c|}
\hline$\rho$ & $\Delta R$ & Control Performance \\
\hline$\rho=0.0001$ (constant) & $\Delta R \leq 7 \%$ & $\begin{array}{l}\text { Stable system and very good control performances } \\
\text { (settling time: } 0.2 \mathrm{~s} \text {.) }\end{array}$ \\
\hline$\rho=0.001$ (constant) & $\Delta R \leq 7 \%$ & $\begin{array}{l}\text { Stable system and very good control performances } \\
\text { (settling time: } 0.2 \mathrm{~s} \text {.) }\end{array}$ \\
\hline$\rho=0.01$ (constant) & $\Delta R \leq 7 \%$ & $\begin{array}{l}\text { Stable system and very good control performances } \\
\text { (settling time: } 0.2 \text {.) }\end{array}$ \\
\hline$\rho=0.1$ (constant) & $\Delta R \leq 8 \%$ & $\begin{array}{l}\text { Stable system but poor control performances } \\
\text { (settling time: under } 2 \mathrm{~s} \text { ) }\end{array}$ \\
\hline$\rho \in\{0.0001,0.1\}$ (variable) & $\Delta R \leq 11 \%$ & $\begin{array}{l}\text { Stable system and very good control performances } \\
\text { (settling time: } 0.5 \mathrm{~s} \text {.) }\end{array}$ \\
\hline
\end{tabular}




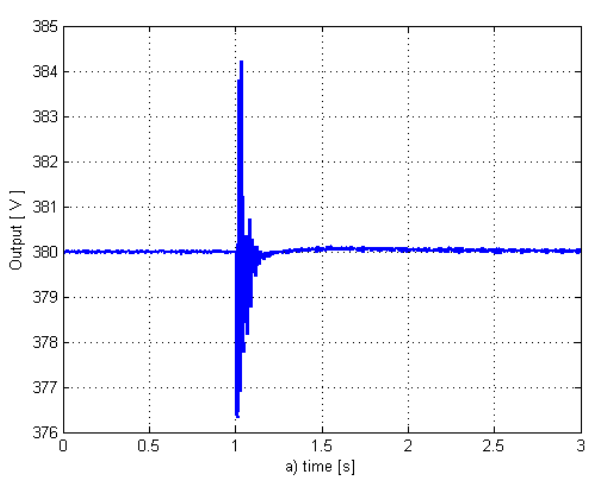

(a)

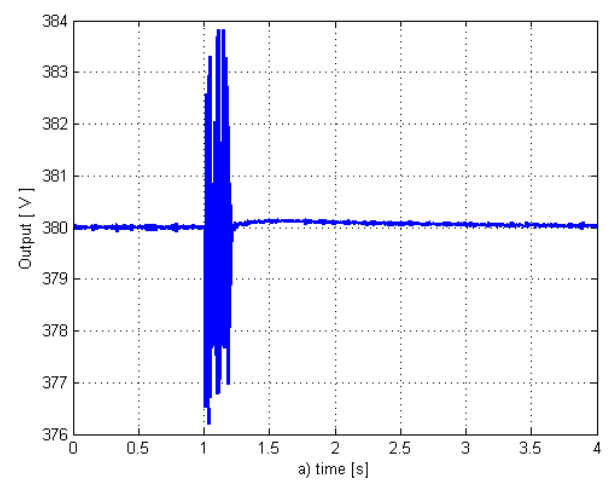

(c)

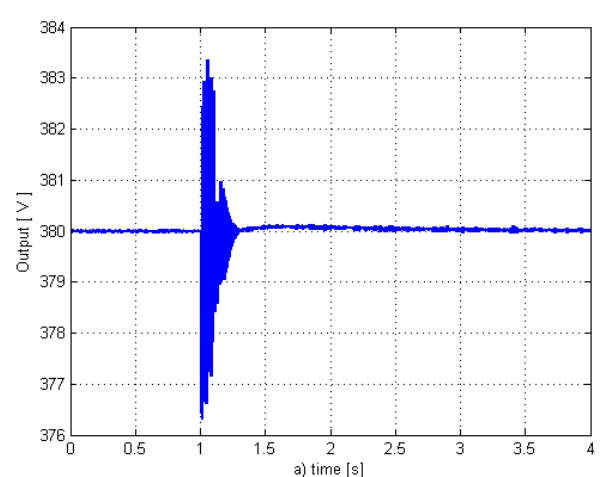

(b)

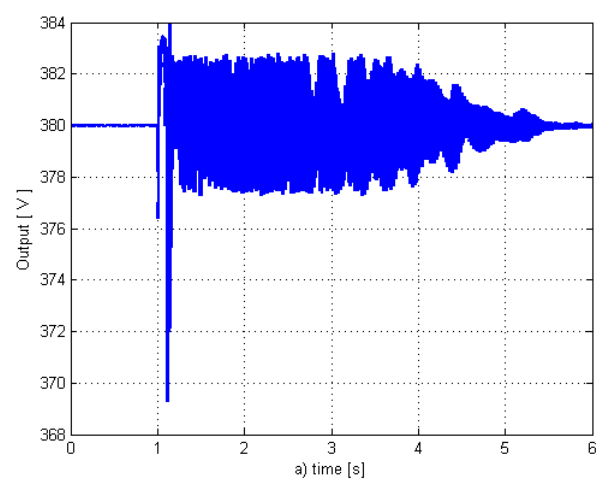

(d)

Figure 6. (a) Controlled output (terminal voltage) $-\rho=0.0001(\Delta R=7 \%)$; (b) Controlled output (terminal voltage) $-\rho=0.001(\Delta R=7 \%)$; (c) Controlled output (terminal voltage) $-\rho=0.01(\Delta R=7 \%)$; (d) Controlled output (terminal voltage) $-\rho=0.1(\Delta R=8 \%)$.

Also, for this disturbance (electrical load) and for small control penalizations ( $\rho \in$ $\{0.0001,0.001,0.01\}$ ), it can be noticed that the maximum allowed disturbance (electrical resistive variation for which the system still remains stable) is the same in all three cases: $\Delta R=7 \%$ (Figure 6a-c). The best control performance is obtained for a small control penalization $(\rho=0.0001$-see Figure 6a). Similar good results are also provided by setting $\rho=0.001$ or $\rho=0.001$ (Figure $6 \mathrm{~b}, \mathrm{c}$ ). For a higher value $\rho=0.1$ and for a slightly increased disturbance $\Delta R=8 \%$ (Figure 6d), the system slowly stabilizes but the control performances are poor (long oscillating regime, long settling time- $5 \mathrm{~s}$ ). Although the system is at last stabilized, the control performance is too weak to be acceptable. Over this value of the disturbance ( $\Delta \mathrm{R}>8 \%$ ), the system becomes unstable due to the excessive control (too weakly penalized).

Therefore, also for this disturbance (load variation), the conclusion is similar to the one reached for the case of a mechanical torque variation:

- A small $\rho$ ensures very good performances, but cannot stabilize a highly disturbed system;

- A high $\rho$ ensures the system stabilization for a higher disturbance, but with the price of an unacceptable degradation of control performance (long oscillating transient regime, long settling time).

Using the proposed tuning mechanism, similar tests were performed for load regimes (generated by connecting/disconnecting an electrical consumer at generator terminals). Thus, taking into consideration a higher electrical load $(\Delta R=11 \%)$, the results are depicted in Figure $7 \mathrm{a}-\mathrm{d}$. It is highlighted that for a constant smaller $\rho$, the maximum value of the disturbance which allowed stabilization of the control system was $\Delta R=7 \%$. 


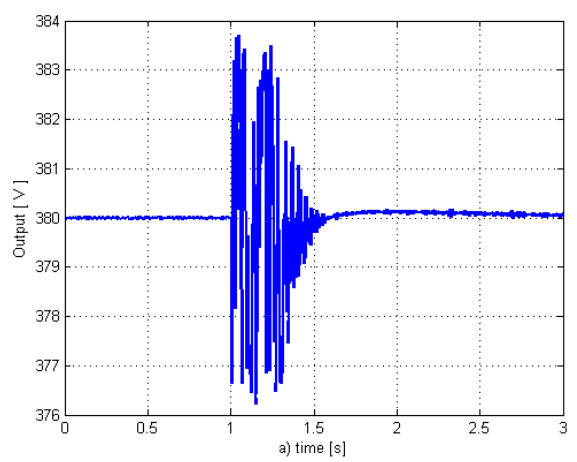

(a)

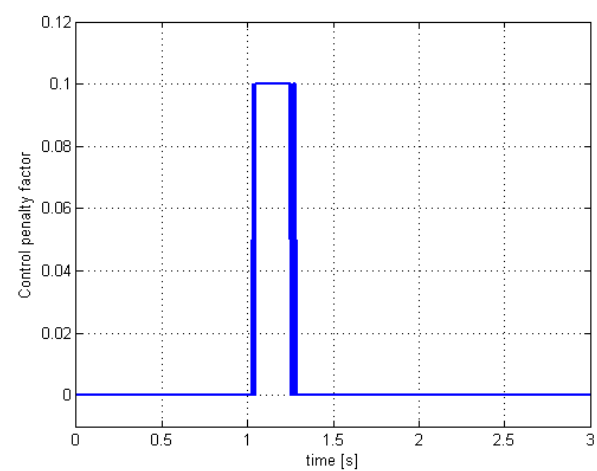

(c)

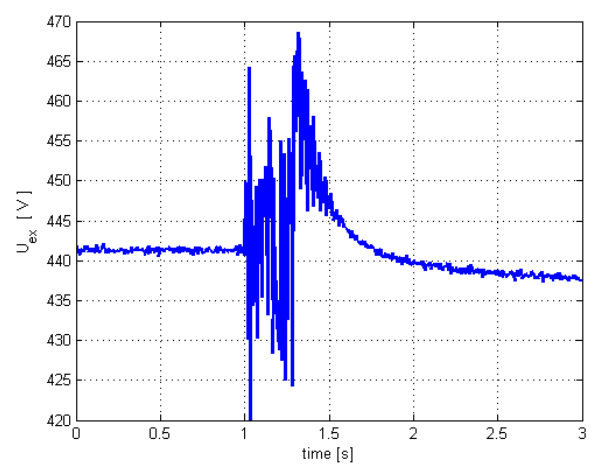

(b)

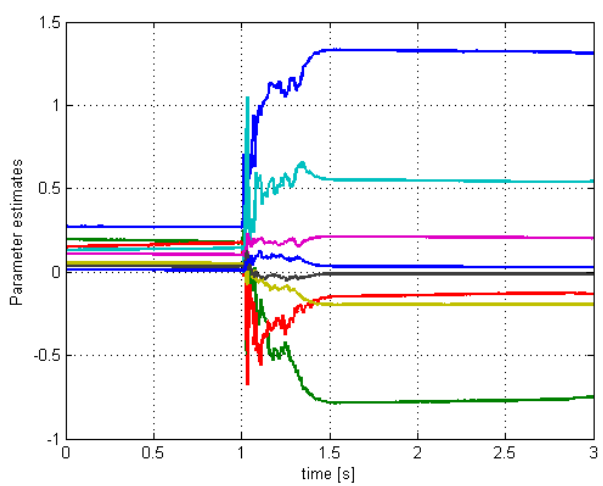

(d)

Figure 7. (a) Controlled output (terminal voltage)—electrical load $(\Delta R=11 \%)-\rho \in\{0.0001,0.1\}$; (b) Controller output (excitation voltage)-( $\Delta R=11 \%), \rho \in\{0.0001,0.1\})$; (c) Variable setting of control penalty factor- $\rho \in\{0.0001,0.1\}(\Delta R=11 \%)$; (d) Parameter estimates.

For this maximum resistive load $\Delta R=11 \%$, the proposed control strategy is still efficient, stabilizing the control system (Figure 7a). A short oscillating regime and a good settling time ( $0.5 \mathrm{~s})$ can be noticed for the controlled output (Figure 7a), the tuning mechanism being triggered for a limited time (Figure 7c) in order to penalize the control (Figure 7b) and thus to stabilize the system.

Therefore, for a load regime, the new control strategy covers an extended value range of disturbance (electrical resistance variation) up to $50 \%$, from $\Delta R=7 \%$ (the maximum allowable load/unload for which a small constant $\rho=0.0001$ still provides good control performance and system stabilization-see Figure 6a) up to $\Delta R=11 \%$ (the maximum load for which the proposed tuning mechanism $\rho \in\{0.0001,0.1\}$ can ensure good performance and can stabilize the system-see Figure 7a).

By analyzing the results of both performed cases (mechanical torque variation and electrical resistive load), it can be concluded that $\rho=0.0001$ is the single constant value of the control penalty factor that simultaneously ensures the best control performance and the control system stability. Unfortunately, for such a constant set value, the maximum variations of the two considered disturbances (for which the system can still be stabilized) are $\Delta T_{m}=16 \%$ and $\Delta R=7 \%$. These two values are considered as benchmarks for assessing the effectiveness of the proposed control strategy, (in terms of system stabilization for a wider value range of disturbances). For larger disturbances, the system can operate stably only using the proposed solution.

Overall, the final conclusion for both types of disturbance (mechanical torque variation and electrical load/unload) is that the proposed control strategy extends the variation range of disturbances by over $50 \%$. For a mechanical torque variation (which can be measured), the solution proposed in [17] offers a better result, allowing a maximum variation of mechanical torque up to $33 \%$, compared with $24 \%$ in this case. As mentioned, without any tuning strategy of the control penalty (so for a constant 
penalty), the maximum allowed variation is only $16 \%$. However, the main advantage of the solution proposed in this paper is that it can also treat the case of unmeasurable perturbation (such as electrical load/unload).

Two additionally case studies are conducted to analyze the performances of the proposed control strategy under action of strong random disturbances, in order to prove the stability and robustness of such a control system.

In the first case (Figure 8), a long sequence of random variations of mechanical torque is considered acting on the system. The controlled output (terminal voltage (Figure 8a)) is stable, proving the control system robustness and good performances of the proposed strategy. Both small and large disturbance can be noticed in Figure $8 b$, and Figure $8 c$ shows the activation cycle of the tuning mechanism that sets the control penalty factor.

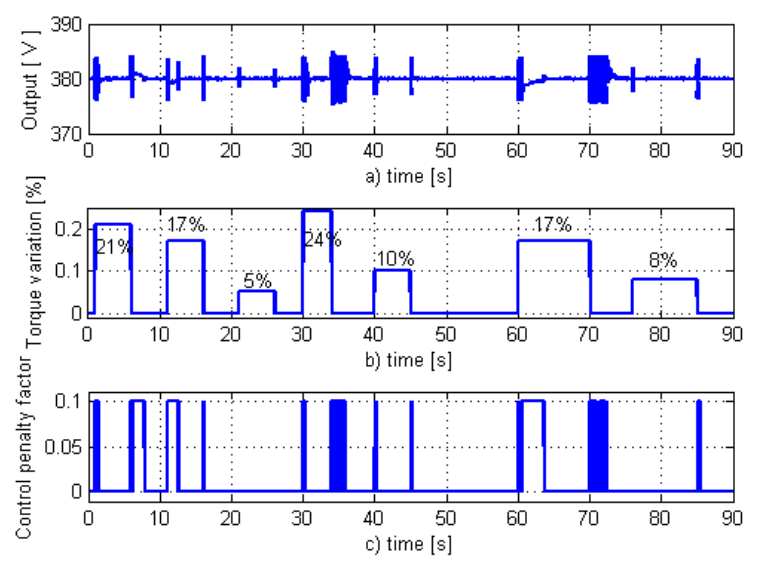

Figure 8. Robustness test-mechanical torque variation: (a) controlled output (terminal voltage); (b) mechanical torque variation (disturbance); (c) variable setting of control penalty factor.

Similarly, for the second case (Figure 9), a long sequence of random electrical load/unload is considered as acting on the controlled process (Figure 9b). A stable system output can be noticed in Figure 9a under control of the proposed tuning mechanism which adjusts the penalty factor $\rho$ (Figure 9c). Again, the performed test proves the stability and robustness of the control system, also for this type of disturbance. Thus, even under stress conditions produced by various random disturbances, the performed case studies demonstrate the validity of the proposed self-tuning mechanism of control penalty factor, improving the performances of such a minimum-variance controller by significantly extending the value range of disturbances (acting on the controlled process) for which the control system remains stable and operational.

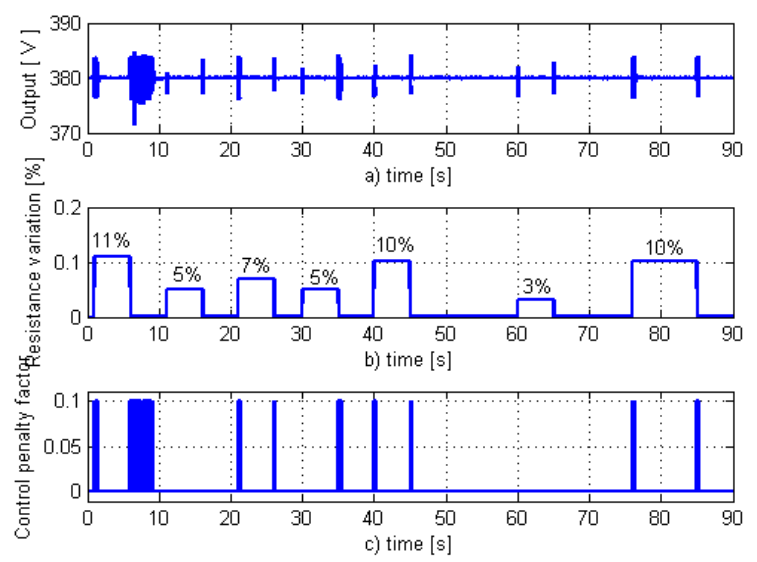

Figure 9. Robustness test-electrical load/unload: (a) controlled output (terminal voltage); (b) electrical resistance variation (disturbance); (c) variable setting of control penalty factor. 
A comparative study between the proposed self-tuning control strategy (involving a variable control penalty), a classical PID controller (tuned for a fixed operating point), an adaptive PID control system and, of course, the classical minimum-variance control law (with a constant control penalty) was conducted in the following.

First, we consider a classical PID controller. As already mentioned in Chapter 1, an induction generator (integrated into a wind energy conversion system) is a nonlinear process, described by a seventh order nonlinear model (Park's equations) [20-24]. The control of a nonlinear plants using a linear controller is a difficult task (and a classical PID controller is a linear controller). Although a PID controller certainly has advantages for linear systems, this controller is not suitable for high-order nonlinear systems $[17,33,34]$. Also, the mechanical torque variation $\left(\Delta T_{m}\right)$ is a measurable external disturbance, which does not directly act on the controlled output (terminal voltage), but rather as an internal perturbation caused by a variation of a process model parameter ( $T_{m}$-see Appendix A). Therefore, the variation of the mechanical torque (considered as a Park's model parameter) leads to a change of the operating point along the nonlinear characteristic of the process. For linear plants, there are many tuning methods for a PID controller (Kessler, Zigler-Nichols, Cohen-Coon, etc.) described by the technical literature [35]. However, an accurate tuning of a PID controller becomes very difficult for a highly nonlinear system. For this case, the tuning was performed through empirical attempts.

The next test scenario considers two study cases for a mechanical torque variation: the case of a small perturbation $\Delta T_{m}=10 \%$ (Figure 10a,b) and the case of a high perturbation $\Delta T_{m}=24 \%$ (Figure 11a-d), as the maximum disturbance allowed only by using the proposed self-tuning strategy of control penalty factor $(\rho)$.

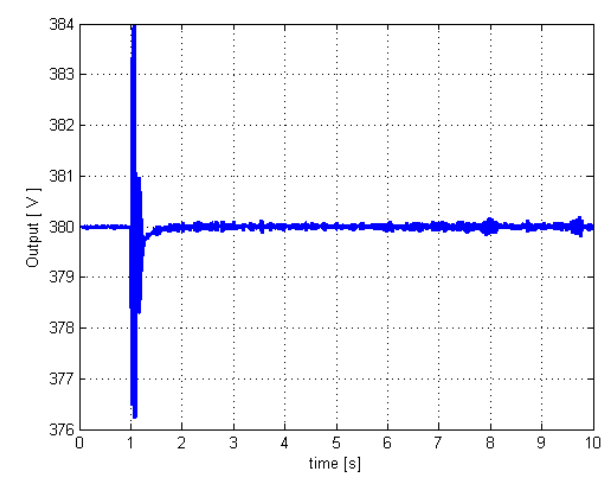

(a)

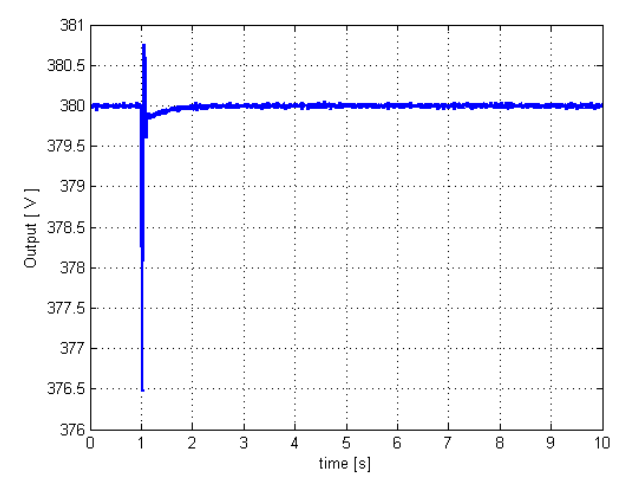

(b)

Figure 10. (a) Controlled output-minimum variance controller $\left(\left(\rho=0.1, \Delta T_{m}=10 \%\right)\right.$, small disturbance); (b) Controlled output—classical PID controller $\left(\left(\Delta T_{m}=10 \%\right)\right.$, small disturbance).

For a small perturbation, by comparing the results obtained by using a self-tuning minimum variance controller with constant control penalty (Figure 10a) and a classical PID controller (Figure 10b), there can be noticed relatively comparable performances. The overshoot is smaller for the PID control system, while the settling time is smaller for the classical minimum variance control. It is mentioned that the same response (see Figure 10a) is obtained also by using the new proposed self-tuning mechanism, which does not activate an increase of the control penalty factor for a small disturbance, so $\rho=0.0001$. Therefore, for small disturbances, the control performances are comparable for all these analyzed control systems. 


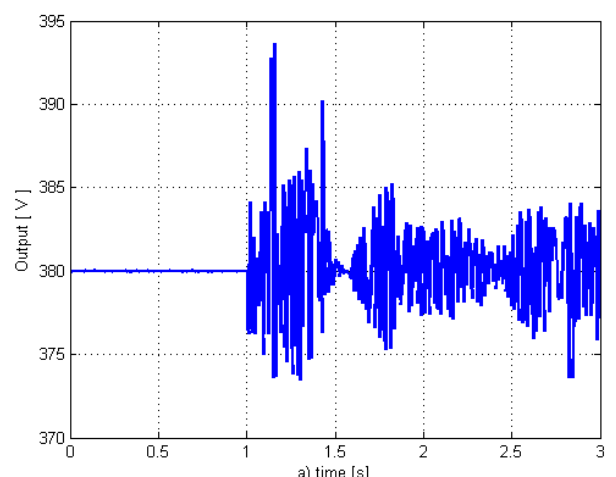

(a)

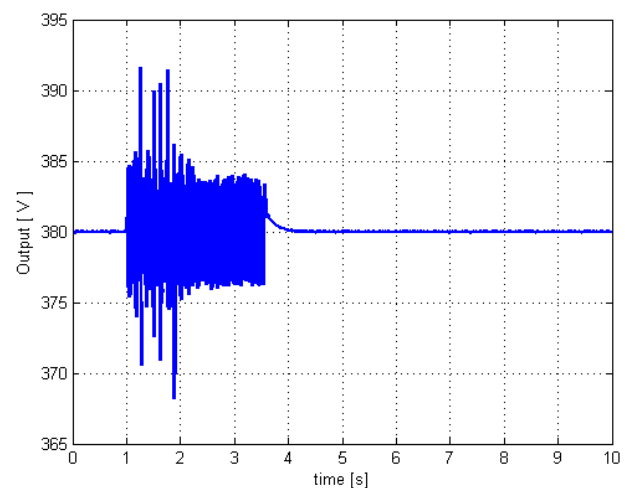

(c)

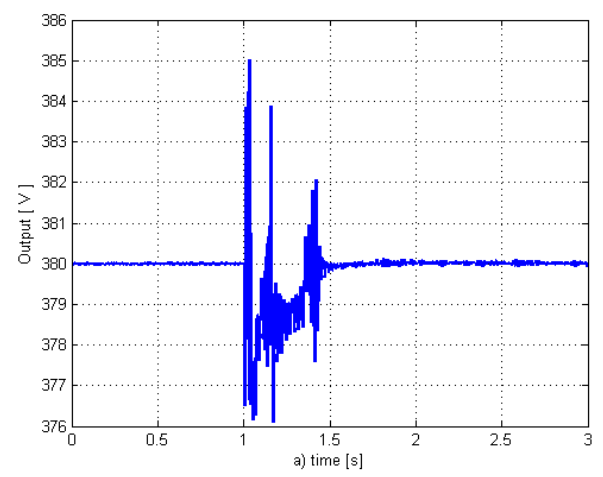

(b)

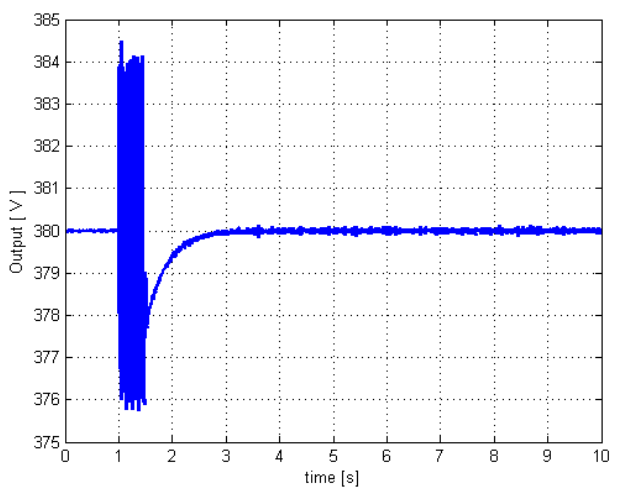

(d)

Figure 11. (a) Controlled output—classical minimum variance controller $\left(\left(\rho=0.1, \Delta T_{m}=24 \%\right)\right.$, high disturbance)—unstable system; (b) Controlled output—-the proposed minimum variance controller ( $\rho \in\left\{0.0001,0.1, \Delta T_{m}=24 \%\right.$ - high disturbance); (c) Controlled output-classical PID controller $\left(\Delta T_{m}=24 \%\right.$ - high disturbance); (d) Controlled output-adaptive PID controller retuned $\left(\Delta T_{m}=24 \%\right.$ high disturbance).

By considering a high disturbance, as we already mentioned, even for a high control penalty factor ( $\rho=0.1$ ), the classical minimum variance control system (with constant control penalty) stabilizes the system only for a maximum disturbance $\Delta T_{m}=19 \%$ (see Figure 3f) and cannot stabilize the system for a higher disturbance $T_{m}=24 \%$ (Figure 11a). Thus, for this situation, the proposed self-tuning control penalty strategy must be taken into consideration (Figure 11b). In this case, for the same already tuned parameters of the PID controller, the change of the operating point leads to poor performances of the PID control (see comparatively the settling times and the overshoots in Figure 11b,c). Therefore, a retuning of the PID controller is required (and this task is not easy at all) and a new set of PID parameters were tuned corresponding to this new functioning point. The result depicted in Figure 11d proves that a proper retuning can considerably improve the PID control performance. Although the control system response presented in Figure 11d is relatively good, by comparison with the one from Figure $11 \mathrm{~b}$, it can be noticed that the settling time is much reduced for the case of the proposed solution. As a conclusion, it can be stated that the performance of a classical PID controller is lower than that of the proposed self-tuning minimum variance controller (with variable control penalty factor), due to the nonlinearity of the controlled process. However, a self-adapting PID controller can be an alternative solution. Even so, a self-tuning minimum variance controller has the advantage of using a reliable and well-known parameter estimation method (Recursive Least Squares) that can automatically solve the issue of self-tuning and can adapt the solution to any other process. Similar tests were performed also for the case of a load/unload disturbance with the same conclusion.

The conclusion is that the proposed adaptive minimum-variance control system (with variable control penalty factor) ensures good performances, comparable to those of other adaptive control 
systems and far superior to a non-adaptive control system (tuned for a fixed operating point). The proposed solution can improve the classical minimum-variance control strategy, allowing the extension of the stability and operability range of a highly disturbed process (in this case due to mechanical torque variation, load/unload), while ensuring good performances, comparable to other adaptive nonlinear control systems. The main advantage of the proposed solution consists in its flexibility and easy adaptation to other considered process. As a future work, a practical validation of the proposed control strategy is taken into consideration and the obtained experimental results can be the subject of a new paper.

\section{Conclusions}

An improved structure of a minimum-variance controller is proposed in this paper, by designing a self-tuning mechanism of the control penalty factor (as a contribution), in order to extend the allowed value range of disturbances, and therefore, implicitly to extend the operating range of the control system. The value of this parameter $(\rho)$ decisively influences the minimum variance control system performance. As a novelty, unlike the classic minimum-variance control, which involves a constant value of $\rho$, the proposed solution supposes a real-time tuning of this controller parameter, based on system output error measurement. The tuning mechanism implements a bipositional switching strategy that considers a sharp hysteresis loop to adjust $\rho$. Therefore, when the system output error exceeds a certain threshold, $\rho$ is set to a higher value, improving the system robustness against disturbances. However, because the best control performances are obtained for a small value of $\rho$, this high-level penalty of control is maintained only at the beginning of the transient regime that occurs due to external disturbances. When the output error drops below a certain threshold, the proposed mechanism resets the control penalty factor to the previous small value (constant in the steady state regime), which ensures the best control system performance. Such a tuning strategy is an efficient solution to stabilize a highly disturbed system, while ensuring good control performance. The performed studies proved that a constant value of $\rho$ (small or high) allows a limited operating range, the control system being destabilized by disturbances that exceed certain levels. By using this strategy (involving a variable control penalty factor), the process operating range can be extended and the control system can withstand higher disturbances in stable operating conditions.

The proposed strategy was validated for the case of an induction generator integrated into a wind energy conversion system and disturbed by two major external perturbations: mechanical torque variations and terminal electrical load/unload. Operating under the action of strong disturbances (wind gusts, electrical load variations), the extension of safe operating range (thus avoiding the system disengagement) is an important goal of such an energy conversion system. The results of the performed tests are relevant, proving the possibility of a stable system operating regime, even under the action of higher disturbances with over $50 \%$ above the limit values allowed if $\rho$ would be constant. Although the tuning strategy was validated for the particular case of an induction generator, the control solution could be used for other highly disturbed processes, especially when the perturbations are difficult (or even impossible) to measure.

Author Contributions: I.F. and F.D. performed the design of the proposed control strategy. I.S. and A.A. performed the simulation and analyzed the obtained results. I.F. and I.S. elaborated the case studies. All authors have structured and approved the final manuscript.

Funding: This research received no external funding.

Conflicts of Interest: The authors declare no conflict of interest. 


\section{Appendix A}

Model of the dual fed induction generator (DFIG) connected to a power system (PS) through a long transmission line [10]

$$
\begin{aligned}
& R_{1} i_{d 1}+L_{d 1} \frac{d}{d t}\left(i_{d 1}\right)+L_{d 21} \frac{d}{d t}\left(i_{d 2}\right)+L_{1 h} \frac{d}{d t}\left(i_{d 3}\right)-\omega_{1}\left(L_{q 1} i_{q 1}+L_{q 21} i_{q 2}+L_{1 h} i_{q 3}\right)-u_{e x}=0 \\
& R_{1} i_{q 1}+L_{q 1} \frac{d}{d t}\left(i_{q 1}\right)+L_{q 21} \frac{d}{d t}\left(i_{q 2}\right)+L_{1 h} \frac{d}{d t}\left(i_{q 3}\right)+\omega_{1}\left(L_{d 1} i_{d 1}+L_{d 21} i d_{2}+L_{1 h} i_{d 3}\right)=0 \\
& -\left(R_{2}+R_{C}\right) i_{d 2}-L_{d 12} \frac{d}{d t}\left(i_{d 1}\right)-L_{d 2} \frac{d}{d t}\left(i_{d 2}\right)-L_{1 h} \frac{d}{d t}\left(i_{d 3}\right)+\omega_{1}\left(L_{q 12} i_{q 1}+L_{q 2} i_{q 2}+L_{1 h} i_{q 3}\right)=0 \\
& -\left(R_{2}+R_{C}\right) i_{q 2}-L_{q 12} \frac{d}{d t}\left(i_{q 1}\right)-L_{q 2} \frac{d}{d t}\left(i_{q 2}\right)-L_{1 h} \frac{d}{d t}\left(i_{q 3}\right)-\omega_{1}\left(L_{d 12} i_{d 1}+L_{d 2} i_{d 2}+L_{1 h} i_{d 3}\right)=0 \\
& R_{3} i_{d 3}+L_{1 h} \frac{d}{d t}\left(i_{d 1}\right)+L_{1 h} \frac{d}{d t}\left(i_{d 2}\right)+L_{d 3} \frac{d}{d t}\left(i_{d 3}\right)-\left(\omega_{1}-\omega\right)\left(L_{1 h} i_{q 1}+L_{1 h} i_{q 2}+L_{q 3} i_{q 3}\right)=0 \\
& R_{3} i_{q 3}+L_{1 h} \frac{d}{d t}\left(i_{q 1}\right)+L_{1 h} \frac{d}{d t}\left(i_{q 2}\right)+L_{q 3} \frac{d}{d t}\left(i_{q 3}\right)+\left(\omega_{1}-\omega\right)\left(L_{1 h} i_{d 1}+L_{1 h} i_{d 2}+L_{d 3} i_{d 3}\right)=0 \\
& \frac{d \omega}{d t}=\left(p \cdot L_{1 \mathrm{~h}} \cdot\left(i_{q 1} \cdot i_{d 3}+i_{d 3} \cdot i_{q 2}-i_{d 1} \cdot i_{q 3}-i_{q 3} \cdot i_{d 2}\right)+T_{m}\right) \cdot p / J \\
& i_{d}=G_{1} V_{d}-B_{1} V_{q}-G_{2} V_{b} \sin (\delta)+B_{2} V_{b} \cos (\delta) \\
& i_{q}=G_{1} V_{q}+B_{1} V_{d}-G_{2} V_{b} \cos (\delta)-B_{2} V_{b} \sin (\delta) \\
& i_{d}=i_{d 2} \text { and } i_{q}=i_{q 2} \\
& V_{d}=\frac{1}{B_{1}^{2}+G_{1}^{2}}\left[i_{d} G_{1}+i_{q} B_{1}+\left(G_{1} G_{2}+B_{1} B_{2}\right) V_{b} \sin (\delta)+\left(B_{1} G_{2}-B_{2} G_{1}\right) V_{b} \cos (\delta)\right] \\
& V_{q}=\frac{1}{B_{1}^{2}+G_{1}^{2}}\left[i_{q} G_{1}-i_{d} B_{1}+\left(B_{1} G_{2}-B_{2} G_{1}\right) V_{b} \sin (\delta)+\left(G_{1} G_{2}+B_{1} B_{2}\right) V_{b} \cos (\delta)\right] \\
& V_{t}=\sqrt{V_{d}^{2}+V_{q}^{2}} \\
& G_{1}=\frac{R_{L}}{R_{L}^{2}+X_{L}^{2}}+\frac{R_{C}}{R_{C}^{2}+X_{C}^{2}} \\
& B_{1}=-\frac{X_{L}}{R_{L}^{2}+X_{L}^{2}}+\frac{X_{C}}{R_{C}^{2}+X_{C}^{2}} \\
& G_{2}=\frac{R_{L}}{R_{L}^{2}+X_{L}^{2}}=\text { constant } \\
& B_{2}=\frac{X_{L}}{R_{L}^{2}+X_{L}^{2}}=\text { constant }
\end{aligned}
$$

The following notations were used in equations: $\omega$ is the rotation speed; $\omega_{1}$ is the synchronous speed; $T_{m}$ is the mechanical torque; $i_{d 1}, i_{d 2}, i_{d 3}, i_{q 1}, i_{q 2}, i_{q 3}$ are the currents projections on $d$ - $q$ axis, for each of the three windings: stator excitation, stator load and rotor; $J$ is the inertia moment; $u_{e x}$ is the excitation voltage; $R_{1}$ is the stator excitation winding resistance; $R_{2}$ is the stator load winding resistance; $R_{3}$ is the rotor winding resistance; $L_{d 1}, L_{q 1}$, are the $d-q$ axis inductance projections of the stator excitation winding; $L_{d 2}, L_{q 2}$ are the $d-q$ axis inductance projections of the stator load winding; $L_{d 3}, L_{q 3}$ are the $d-q$ axis inductance projections of the rotor winding; $L_{d 12}, L_{d 21}, L_{q 12}, L_{q 21}, L_{1 h}$ are the leakage/mutual inductances; $p$ is the number of pole pairs; $Y_{1}=G_{1}+j B_{1}=Y_{L}+Y_{C}$ is the transmission line admittance seen at the generator terminals $\left(G_{1}, B_{1}\right.$,-conductance, susceptance)—see Figure A1; $Y_{2}=G_{2}+j B_{2}$ is the 
transfer admittance between the generator and the power grid $\left(G_{2}, B_{2}\right.$ - conductance, susceptance $)-$ see Figure $\mathrm{A} 1 ; R_{L}, R_{C}$ are the line and consumer resistances; $X_{L}, X_{C}$ are the line and consumer reactances; $i_{d}$ and $i_{q}$ are the terminals current on $d-q$ axis; $V_{d}$ and $V_{q}$ are voltage projections of stator load winding on $d-q$ axis (and also projections of $V_{t}$ generator terminals voltage on $d-q$ axis); $V_{t}=\sqrt{V_{d}^{2}+V_{q}^{2}}$ is the the generator terminal voltage (effective value).

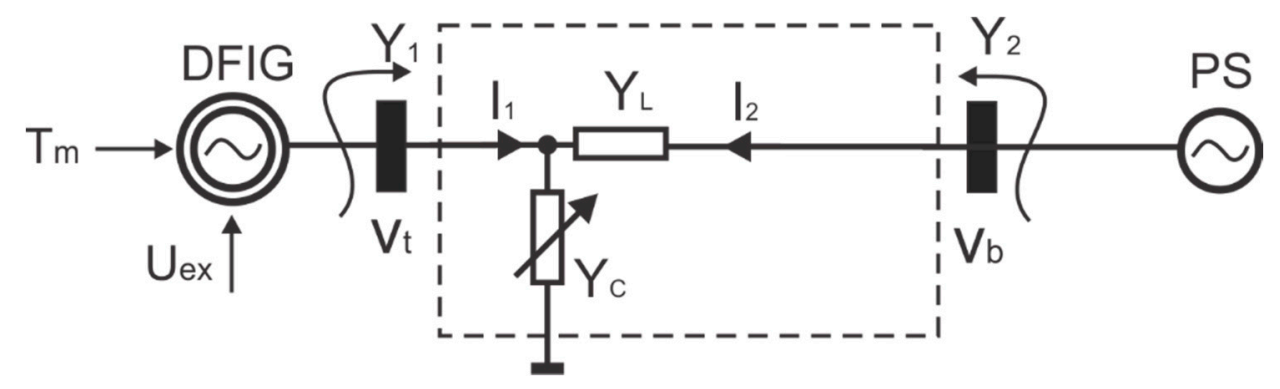

Figure A1. Induction generator with local electrical load (connected to a power system).

There is considered an experimental double fed induction machine (a prototype) with the following main parameters (index $N$ denoting rated values): $P_{N}=1.5[\mathrm{~kW}], U_{N}=230 / 400[\mathrm{~V}], I_{N}=2.06 / 3.57[\mathrm{~A}]$, $n_{0}=1500[\mathrm{rpm}], \cos \phi_{N}=0.776, s_{N}=5.79 \%, p=2$ (number of pair poles), $L_{d 21}=L_{d 12}=L_{q 21}=L_{q 12}=$ $0.333[H], L_{1 h}=0.318[H], L_{q 1}=0.334[H], L_{q 2}=0.334[H], L_{q 1}=0.331[H], L_{d 1}=0.334[H], L_{d 2}=0.334$ $[H], L_{d 3}=0.334[H], R_{1}=16[\Omega], R_{2}=16[\Omega], R_{3}=4[\Omega], J=0.00415\left[\mathrm{~kg} \cdot \mathrm{m}^{2}\right]$. The stator windings $w_{1}$ and $w_{3}$ are placed in the same stator cuts; the $w_{2}$ winding is spatially lagged with 90 electrical degrees in relation with the $w_{1}$ winding.

The Equations (1)-(17) (practically, a seventh order nonlinear model) completely describe the behavior of interconnected systems (induction generator, long transmission line, local electrical consumer and power system). The model input is the excitation voltage $u_{e x}$ and the generator terminals voltage $V_{t}$ is the process output. The disturbances acting on the controlled process are the mechanical torque $T_{m}$ (active power load) and the electrical consumer resistance $R_{c}$, affecting the admittance seen at the generator terminals and allowing simulation of load/unload regimes by connecting or disconnecting local consumers.

\section{References}

1. Benosman, M. Learning-Based Adaptive Control. In An Extremum Seeking Approach-Theory and Applications, Butterworth-Heinemann; Elsevier: Amsterdam, Netherlands, 2017.

2. Aström, K.J.; Kumarb, P.R. Control: A perspective. Automatica 2014, 1, 3-43. [CrossRef]

3. Bobal, V.; Böhm, V.; Fessl, J.; Machácek, J. Digital Self-Tuning Controllers: Algorithms, Implementation and Applications (Advanced Textbooks in Control and Signal Processing); London Limited, Ed.; Springer: London, UK, 2005.

4. Ishchenko, A.; Myrzik, J.M.A.; Kling, W.L. Linearization of Dynamic Model of Squirrel-Cage Induction Generator Wind Turbine. In Proceedings of the IEEE Power Engineering Society General Meeting, Tampa, FL, USA, 24-28 June 2007; pp. 1-8.

5. Ky, M.V. Optimal Discrete Control Theory: The Rational Function Structure Model; AuLac Technologies Inc.: Ottawa, ON, Canada, 2007.

6. Zou, Y.; Elbuluk, M.; Sozer, Y. A Complete Modeling and Simulation of Induction Generator Wind Power Systems. In Proceedings of the IEEE Industry Applications Society Annual Meeting (IAS), Houston, TX, USA, 3-7 October 2010; pp. 1-8. [CrossRef]

7. Filip, I.; Szeidert, I. Adaptive fuzzy PI controller with shifted control singletons. Expert Syst. Appl. 2016, 54, 1-12. [CrossRef]

8. Filip, I.; Vasar, C. About Initial Setting of a Self-Tuning Controller. In Proceedings of the 4th International Symposium on Applied Computational Intelligence and Informatics, SACI, Timisoara, Romania, 17-18 May 2007; pp. 251-256. [CrossRef] 
9. Meng, Z.; Jiang, J.; Dang, D. Evolutionary Algorithm for Zero-One Constrained Optimization Problems Based on Objective Penalty Function. In Proceedings of the 9th International Conference on Computational Intelligence and Security, Nanning, China, 11-14 December 2010; pp. 132-136. [CrossRef]

10. Filip, I.; Mihet-Popa, L.; Vasar, C.; Prostean, O.; Szeidert, I. Considerations Regarding the Design of a Minimum Variance Control System for an Induction Generator. Electronics 2019, 8, 532. [CrossRef]

11. Filip, I.; Prostean, O.; Szeidert, I.; Vasar, C. Consideration Regarding the Convergence and Stability of an Adaptive Self-Tuning Control System. In Proceedings of the 5th IEEE International Conference on Computational Cybernetics, Gammarth, Tunisia, 19-21 October 2007; pp. 75-79. [CrossRef]

12. Filip, I.; Szeidert, I. Givens Orthogonal Transformation-Based Estimator Versus RLS Estimator-Case Study for an Induction Generator Model; Soft Computing Applications, Advances in Intelligent Systems and Computing, 357; Springer: Cham, Switzerland, 2015; pp. 1287-1299. [CrossRef]

13. Rohwer, C.M.; Angeletti, F.; Touchette, H. Convergence of large-deviation estimators. Phys. Rev. E 2015, 5, 2104. [CrossRef] [PubMed]

14. Zhang, S.; Zhang, J. An RLS Algorithm with Evolving Forgetting Factor. In Proceedings of the 7th International Workshop on Signal Design and its Applications in Communications (IWSDA), Bengaluru, India, 14-18 September 2015; pp. 24-27.

15. Filip, I.; Szeidert, I.; Prostean, O. Mathematical Modelling and Numerical Simulation of the Dual Winded Induction Generator's Operating Regimes; Soft Computing Applications, Advances in Intelligent Systems and Computing, 357; Springer: Cham, Switzerland, 2015; pp. 1161-1170. [CrossRef]

16. Filip, I.; Szeidert, I. Tuning the control penalty factor of a minimum variance adaptive controller. Eur. J. Control 2017, 37, 16-26. [CrossRef]

17. Filip, I.; Vasar, C.; Prostean, O.; Szeidert, I. Self-tuning strategy for a minimum variance control system of a highly disturbed process. Eur. J. Control 2019, 46, 49-62. [CrossRef]

18. Filip, I.; Prostean, O.; Szeidert, I.; Vasar, C. An Improved Structure of an Adaptive Excitation Control System Operating under Short-Circuit. Adv. Electr. Comput. Eng. 2016, 16, 43-50. [CrossRef]

19. Lavretsky, E.; Wise, K. Robust and Adaptive Control: With Aerospace Applications (Advanced Textbooks in Control and Signal Processing); London Limited, Ed.; Springer: London, UK, 2012.

20. Zribi, M.; Alrifai, M.; Rayan, M. Sliding Mode Control of a Variable-Speed Wind Energy Conversion System Using a Squirrel Cage Induction Generator. Energies 2017, 10, 604. [CrossRef]

21. Ni, K.; Hu, Y.; Liu, Y.; Gan, C. Performance Analysis of a Four-Switch Three-Phase Grid-Side Converter with Modulation Simplification in a Doubly-Fed Induction Generator-Based Wind Turbine (DFIG-WT) with Different External Disturbances. Energies 2017, 10, 706. [CrossRef]

22. Xypolytou, E.; Gawlik, W.; Zseby, T.; Fabini, J. Impact of Asynchronous Renewable Generation Infeed on Grid Frequency: Analysis Based on Synchrophasor Measurements. Sustainability 2018, 10, 1605. [CrossRef]

23. Filip, I.; Szeidert, I.; Prostean, O.; Vasar, C. Issues Regarding the Tuning of a Minimum Variance Adaptive Controller; Soft Computing Applications, Advances in Intelligent Systems and Computing, 633; Springer: Cham, Switzerland, 2018; pp. 70-77. [CrossRef]

24. Mikles, J.; Fikar, M. Process Modelling, Identification, and Control; Springer: Berlin/Heidelberg, Germany, 2007.

25. Huang, X.F.; Tang, X.F.; Deng, X.; Wang, X.J. The large deviation for the least squares estimator of nonlinear regression model based on WOD errors. J. Inequalities Appl. 2016, 125, 2-11. [CrossRef]

26. Zidane, Z.; Lafkih, M.A.; Ramzi, M.; Abounada, A. Adaptive Minimum Variance Control of a DC Motor. In Proceedings of the 18th Mediterranean Conference on Control \& Automation Congress, Marrakech, Morocco, 23-25 June 2010; pp. 1-4. [CrossRef]

27. Hou, G.; Hu, G.; Sun, R.; Zhang, J. Application of Self-Tuning Control Based on Generalized Minimum Variance Method in Evaporator for ORCS. In Proceedings of the International Conference on Advanced Mechatronic Systems, Zhengzhou, China, 11-13 August 2011; pp. 202-206.

28. Zhang, H.; Liu, D.; Luo, Y.; Wang, D. Adaptive Dynamic Programming for Control. Algorithms and Stability, 2013th ed.; Springer: Cham, Switzerland, 2013. [CrossRef]

29. Zachariah, K.J.; Finch, J.W.; Farsi, M. Multivariable Self-Tuning Control of a Turbine Generator System. IEEE Trans. Energy Convers. 2009, 24, 406-414. [CrossRef]

30. Farias, E.R.C.; Cari, E.P.T.; Erlich, I.; Shewarega, F. Online Parameter Estimation of a Transient Induction Generator Model Based on the Hybrid Method. IEEE Trans. Energy Convers. 2018, 33, 1529-1538. [CrossRef] 
31. Wu, D.; Song, J.; Shen, Y. Variable Forgetting Factor Identification Algorithm for Fault Diagnosis of Wind Turbines. In Proceedings of the Chinese Control and Decision Conference (CCDC), Yinchuan, China, 28-30 May 2016; pp. 1895-1900. [CrossRef]

32. Masayoshi, D.; Kazuhisa, N.; Yutaka, O. Applying Generalized Minimum Variance Control for Ship's Steering Control System. In Proceedings of the SICE Annual Conference, Taipei, Taiwan, 18-21 August 2010; pp. 477-483.

33. Nivetha, J.; Vijayan, V. Design of Tunable Method of PID Controller for Conical Tank System. In Proceedings of the 5th IEEE International Conference on Computation of Power, Energy, Information and Communication, Melmaruvathur, India, 20-21 April 2016; pp. 251-254.

34. Gouadria, F.; Sbita, L.; Sigrimis, N. Comparison between Self-Tuning Fuzzy PID and Classic PID Controllers for Greenhouse System. In Proceedings of the Conference on Green Energy and Conversion Systems, Hammamet, Tunisia, 23-25 March 2017.

35. O'Dwyer, A. Handbook of PI and PID Controller Tuning Rules; Imperial College Press: London, UK, 2006.

(C) 2020 by the authors. Licensee MDPI, Basel, Switzerland. This article is an open access article distributed under the terms and conditions of the Creative Commons Attribution (CC BY) license (http://creativecommons.org/licenses/by/4.0/). 